\title{
Vertex-Disjoint Simple Paths of Given Homotopy in a Planar Graph
}

\author{
A. FRANK AND A. SCHRIJVER
}

\begin{abstract}
We characterize the existence of pairwise vertex-disjoint simple paths $P_{1}, \ldots, P_{k}$ of prescribed homotopy in a given planar graph when all end points of the paths are at the "holes" in the plane. Moreover, we give a polynomial-time algorithm for finding these paths, if they exist. Our methods are polyhedral and make use of the ellipsoid method and of considering a fractional solution to the packing problem.
\end{abstract}

\section{The theorem}

We prove the following theorem, conjectured by L. Lovász and P. D. Seymour.

THEOREM. Let $G=(V, E)$ be a planar graph, embedded in $\mathbb{R}^{2}$, let $I_{1}, \cdots, I_{p}$ be (the interiors of) some of its faces (including the unbounded face), and let $P_{1}, \ldots, P_{k}$ be paths in $G$, each with end points on the boundary of $I_{1} \cup \cdots \cup I_{p}$. Then there exist pairwise vertex-disjoint simple paths $\widetilde{P}_{1}, \ldots, \widetilde{P}_{k}$ in $G$ so that $\widetilde{P}_{i}$ is homotopic to $P_{i}$ in $\mathbb{R}^{2} \backslash\left(I_{1} \cup \cdots \cup I_{p}\right)$ for $i=1, \ldots, k$ if and only if

(i) there are pairwise disjoint simple curves $C_{1}, \ldots, C_{k}$ in $\mathbb{R}^{2} \backslash\left(I_{1} \cup \cdots \cup I_{p}\right)$ such that $C_{i}$ is homotopic to $P_{i}$ in $\mathbb{R}^{2} \backslash\left(I_{1} \cup \cdots \cup I_{p}\right)$ for $i=1, \ldots, k$;

(ii) for each curve $D:[0,1] \rightarrow \mathbb{R}^{2} \backslash\left(I_{1} \cup \cdots \cup I_{p}\right)$ with $D(0), D(1) \in \operatorname{bd}\left(I_{1} \cup \cdots \cup I_{p}\right)$ we have $\operatorname{cr}(G, D) \geq$ $\sum_{i=1}^{k} \min \operatorname{cr}\left(P_{i}, D\right)$;

1980 Mathematics Subject Classification (1985 Revision). Primary 05C38, 57M99.

(C) 1990 American Mathematical Society $1052-1798 / 90 \$ 1.00+\$ .25$ per page 
(iii) if $D_{1}, D_{2}: S_{1} \rightarrow \mathbb{R}^{2} \backslash\left(I_{1} \cup \cdots \cup I_{p}\right)$ is a pair of closed curves with the properties that (a) $D_{1}(1)=D_{2}(1) \notin$ $G$, (b) if $D_{1}$ or $D_{2}$ passes any vertex $v$ of $G$, then for each $i=1, \ldots, k$ there exists a curve homotopic to $P_{i}$ in $\mathbb{R}^{2} \backslash\left(I_{1} \cup \cdots \cup I_{p}\right)$ not passing $v$, (c) for $j=1,2: \operatorname{cr}\left(G, D_{j}\right) \not \equiv \sum_{i=1}^{k} \min \operatorname{cr}\left(P_{i}, D_{j}\right)$ (mod 2), then we have

$$
\operatorname{cr}\left(G, D_{1} \cdot D_{2}\right) \geq 2+\sum_{i=1}^{k} \min \operatorname{cr}\left(P_{i}, D_{1} \cdot D_{2}\right) .
$$

Note that the case $k=1$ amounts to the existence of one simple path of given homotopy.

In the theorem and in the sequel we use the following conventions and terminology.

Graphs and their embeddings. We identify a planar graph $G=(V, E)$ embedded in $\mathbb{R}^{2}$ with its embedding. We consider faces as open regions in $\mathbb{R}^{2}$ and edges as open curves (so without end points). The boundary of .. is denoted by bd(..).

Curves. A curve is a continuous function $C:[0,1] \rightarrow \mathbb{R}^{2}$. A closed curve is a continuous function $C: S_{1} \rightarrow \mathbb{R}^{2}$ (where $S_{1}$ denotes the unit circle in $\mathbb{C}$ ). For closed curves $D_{1}, D_{2}: S_{1} \rightarrow \mathbb{R}^{2}$ with $D_{1}(1)=D_{2}(1)$, the closed curve $D_{1} \cdot D_{2}$ is defined by: $D_{1} \cdot D_{2}(z)=D_{1}\left(z^{2}\right)$ if $\operatorname{Im}(z) \geq 0$ and $D_{1} \cdot D_{2}(z)=$ $D_{2}\left(z^{2}\right)$ if $\operatorname{Im}(z)<0$.

Homotopy. Two curves $C, D:[0,1] \rightarrow X \subseteq \mathbb{R}^{2}$ are called homotopic (in $X$ ), in notation $C \sim D$, if there exists a continuous function $\Phi:[0,1] \times$ $[0,1] \rightarrow X$ so that $\Phi(0, x)=C(x), \Phi(1, x)=D(x), \Phi(x, 0)=C(0)$, and $\Phi(x, 1)=C(1)$ for all $x \in[0,1]$. (Note that this implies $C(0)=D(0)$ and $C(1)=D(1)$.) Two closed curves $C, D: S_{1} \rightarrow X \subseteq \mathbb{R}^{2}$ are called (freely) homotopic (in $X$ ), in notation $C \sim D$, if there exists a continuous function $\Phi:[0,1] \times S_{1} \rightarrow X$ so that $\Phi(0, x)=C(x)$ and $\Phi(1, x)=D(x)$ for all $x \in S_{1}$. (Note that not necessarily $C(1)=D(1)$.)

Paths. A path in graph $G=(V, E)$ is a sequence

$$
\left(v_{0}, e_{1}, v_{1}, \ldots, e_{l}, v_{l}\right)
$$

where $v_{0}, \ldots, v_{l}$ are vertices and $e_{1}, \ldots, e_{l}$ are edges, so that $e_{i}$ connects $v_{i-1}$ and $v_{i}(i=1, \ldots, l)$. The path is simple if $v_{0}, \ldots, v_{l}$ are all distinct. Two paths are vertex-disjoint if they do not have a vertex in common. When $G$ is embedded in $\mathbb{R}^{2}$, we identify a path in the obvious way with any curve following this path in the embedding. (That is, we identify (2) with any 
curve $P:[0,1] \rightarrow \mathbb{R}^{2}$ so that $P(i / l)=v_{i}$ for $i=0, \ldots, l$ and $P(x) \in e_{i}$ if $(i-1) / l<x<i / l$.)

Counting intersections. If $C, D:[0,1] \rightarrow \mathbb{R}^{2} \backslash\left(I_{1} \cup \cdots \cup I_{p}\right)$ are curves, where $I_{1}, \ldots, I_{p}$ are faces of a graph $G=(V, E)$ embedded in $\mathbb{R}^{2}$, then

(3) $\operatorname{cr}(C, D):=|\{(x, y) \in[0,1] \times[0,1] \mid C(x)=D(y)\}|$,

$$
\begin{aligned}
& \min \operatorname{cr}(C, D):=\min \left\{\operatorname{cr}(\widetilde{C}, \widetilde{D}) \mid \widetilde{C} \sim C, \widetilde{D} \sim D\left(\text { in } \mathbb{R}^{2} \backslash\left(I_{1} \cup \cdots \cup I_{p}\right)\right)\right\}, \\
& \operatorname{cr}(G, D):=|\{y \in[0,1] \mid D(y) \in G\}|, \\
& \text { if } D \text { is not a constant function, } \\
&:=1, \text { if } D \text { is a constant function. }
\end{aligned}
$$

If $C:[0,1] \rightarrow \mathbb{R}^{2} \backslash\left(I_{1} \cup \cdots \cup I_{p}\right)$ is a curve and $D: S_{1} \rightarrow \mathbb{R}^{2} \backslash\left(I_{1} \cup \cdots \cup I_{p}\right)$ is a closed curve, then

(4) $\operatorname{cr}(C, D):=\left|\left\{(x, y) \in[0,1] \times S_{1} \mid C(x)=D(y)\right\}\right|$,

$$
\begin{aligned}
\min \operatorname{cr}(C, D) & :=\min \left\{\operatorname{cr}(\widetilde{C}, \widetilde{D}) \mid \widetilde{C} \sim C, \widetilde{D} \sim D\left(\text { in } \mathbb{R}^{2} \backslash\left(I_{1} \cup \cdots \cup I_{p}\right)\right)\right\}, \\
\operatorname{cr}(G, D) & :=\left|\left\{y \in S_{1} \mid D(y) \in G\right\}\right| .
\end{aligned}
$$

Crossings. Two (closed) curves $C, D$ are said to cross if there exist $x, y$ so that $C(x)=D(y)$ and there exists a homeomorphism $\phi: \mathbb{R}^{2} \rightarrow \mathbb{R}^{2}$ so that the functions $\phi \circ C$ and $\phi \circ D$ are linear functions in neighbourhoods of $x$ and $y$, respectively, with different angles. In that case, $(x, y)$ is said to give a crossing. If $C$ and $D$ do not cross, they are called noncrossing.

The greater part of this paper consists of proving sufficiency of the conditions (1), which is based on Lemmas 1 and 2 proved in Sections 3 and 4. Lemma 1 is shown with the help of an auxiliary theorem proved in Section 2.

\section{An auxiliary theorem on edge-disjoint paths}

One ingredient for our proof is the following "homotopic flow-cut theorem" ([6]).

HOMOTOPIC FLOW-CUT THEOREM. Let $G=(V, E)$ be a planar graph embedded in $\mathbb{R}^{2}$, let $I_{1}, \ldots, I_{p}$ be some of the faces of $G$ (including the unbounded face), and let $C_{1}, \ldots, C_{k}$ be curves in $\mathbb{R}^{2} \backslash\left(I_{1} \cup \cdots \cup I_{p}\right)$ with end points in $V \cap \operatorname{bd}\left(I_{1} \cup \cdots \cup I_{p}\right)$. Then there exist paths $P_{1}^{1}, \ldots, P_{1}^{t_{1}}, P_{2}^{1}, \ldots$, $P_{2}^{t_{2}}, \ldots, P_{k}^{1}, \ldots, P_{k}^{t_{k}}$ in $G$ and rational numbers $\lambda_{1}^{1}, \ldots, \lambda_{1}^{t_{1}}, \lambda_{2}^{1}, \ldots, \lambda_{2}^{t_{2}}$, 
$\ldots, \lambda_{k}^{1}, \ldots, \lambda_{k}^{t_{k}}>0$ so that

(10) (i) $P_{i}^{j} \sim C_{i}$ in $\mathbb{R}^{2} \backslash\left(I_{1} \cup \cdots \cup I_{p}\right) \quad\left(i=1, \ldots, k ; j=1, \ldots, t_{j}\right)$,

(ii) $\sum_{j=1}^{t_{i}} \lambda_{i}^{j}=1 \quad(i=1, \ldots, k)$,

(iii) $\sum_{i=1}^{k} \sum_{j=1}^{t_{i}} \lambda_{i}^{j} \chi^{P_{i}^{j}}(e) \leq 1 \quad(e \in E)$,

if and only if for each curve $D:[0,1] \rightarrow \mathbb{R}^{2} \backslash\left(I_{1} \cup \cdots \cup I_{p} \cup V\right)$ with $D(1$ $D(1) \in \operatorname{bd}\left(I_{1} \cup \cdots \cup I_{p}\right)$ we have

$$
\operatorname{cr}(G, D) \geq \sum_{i=1}^{k} \min \operatorname{cr}\left(C_{i}, D\right) .
$$

Here, for any path $P$ in $G$ and any edge $e$ of $G, \chi^{P}(e)$ denotes the num? of times $P$ passes $e$.

As our "auxiliary theorem" we derive that under certain circumstances $\lambda_{i}^{j}$ can be taken to be integral.

AUXILIARY THEOREM. Let $G=(V, E)$ be a planar graph embedded $\mathbb{R}^{2}$, let $I_{1}, \ldots, I_{p}$ be some of the faces of $G$ (including the unbounded fac and let $C_{1}, \ldots, C_{k}$ be curves in $\mathbb{R}^{2} \backslash\left(I_{1} \cup \cdots \cup I_{p}\right)$ with end points in $\mathfrak{I}$ $\operatorname{bd}\left(I_{1} \cup \cdots \cup I_{p}\right)$, so that

(i) each $C_{i}$ has only a finite number of self-intersections and no self-crossings;

(ii) each two of the $C_{i}$ have only a finite number of intersections and no crossings;

(iii) each vertex of $G$ either has degree even and is no end point of any $C_{i}$, or has degree 1 and is an end point of exactly one $C_{i}$.

Then there exist pairwise edge-disjoint and pairwise noncrossing paths $P_{1}$, . $P_{k}$ in $G$, without self-crossings and not passing the same edge more $t i$ once, so that $P_{i} \sim C_{i}$ for $i=1, \ldots, k$, if and only if for each ch $D:[0,1] \rightarrow \mathbb{R}^{2} \backslash\left(I_{1} \cup \cdots \cup I_{p} \cup V\right)$ with $D(0), D(1) \in \operatorname{bd}\left(I_{1} \cup \cdots \cup\right.$ we have (11).

[Here paths are called edge-disjoint if they do not have any edge in cc mon.]

Proof. The "if" part is trivial, since for any $D$ in question we have

$$
\operatorname{cr}(G, D) \geq \sum_{i=1}^{k} \operatorname{cr}\left(P_{i}, D\right) \geq \sum_{i=1}^{k} \min \operatorname{cr}\left(C_{i}, D\right) .
$$


To see the "only if" part, suppose that (11) is satisfied for each curve $D$ in question. By the homotopic flow-cut theorem, there exist paths $P_{i}^{j}$ in $G$ and rationals $\lambda_{i}^{j}>0$ (for $i=1, \ldots, k ; j=1, \ldots, t_{i}$ ) satisfying (10). In fact, as the $\lambda_{i}^{j}$ can be written with one common denominator, say $K$, we may assume that $t_{1}=\cdots=t_{k}=K$ and that each $\lambda_{i}^{j}$ is equal to $1 / K$ (this is achieved by replacing each $P_{i}^{j}$ by $K \cdot \lambda_{i}^{j}$ copies of $P_{i}^{j}$ ). Replacing each edge of $G$ by $K$ parallel edges, we obtain a graph $G^{\prime}=\left(V, E^{\prime}\right)$ and pairwise edge-disjoint paths $P_{1}^{1}, \ldots, P_{1}^{K}, \ldots, P_{k}^{1}, \ldots, P_{k}^{K}$ in $G^{\prime}$. Clearly each face of $G$ corresponds to a face of $G^{\prime}$, and we will use the same name for both of them. In particular, $I_{1}, \ldots, I_{p}$ are again faces of $G^{\prime}$.

Claim 1. We may assume that $P_{i}^{j}$ and $P_{i^{\prime}}^{j^{\prime}}$ are noncrossing, if $i \neq i^{\prime}$.

Proof. Suppose we have chosen the paths $P_{1}^{1}, \ldots, P_{k}^{K}$ so that

$$
\sum_{i=1}^{k} \sum_{i^{\prime}=i+1}^{k} \sum_{j=1}^{k} \sum_{j^{\prime}=1}^{K} \text { (number of crossings of } P_{i}^{j} \text { and } P_{i^{\prime}}^{j^{\prime}} \text { ) }
$$

is as small as possible. We must show that this sum is 0 . Indeed, suppose $P_{i}^{j}$ and $P_{i^{\prime}}^{j^{\prime}}$ have a crossing, where $i \neq i^{\prime}$. As $C_{i}$ and $C_{i^{\prime}}$ have no crossings, there exist $x, x^{\prime}, y, y^{\prime} \in[0,1]$ so that $\left(x, x^{\prime}\right) \neq\left(y, y^{\prime}\right), P_{i}^{j}(x)=P_{i^{\prime}}^{j^{\prime}}\left(x^{\prime}\right)$ and $P_{i}^{j}(y)=P_{i^{\prime}}^{j^{\prime}}\left(y^{\prime}\right)$, so that both $\left(x, x^{\prime}\right)$ and $\left(y, y^{\prime}\right)$ give crossings, and so that the $x-y$ part of $P_{i}^{j}$ is homotopic to the $x^{\prime}-y^{\prime}$ part of $P_{i^{\prime}}^{j^{\prime}}$ (cf. [6]). Exchanging these two parts decreases sum (14), contradicting its minimality.

Clearly, we may assume moreover that no $P_{i}^{j}$ has null-homotopic parts.

Now in order to prove our auxiliary theorem we apply induction on the number of edges of $G$ plus the number of faces of $G$ not in $\left\{I_{1}, \ldots, I_{p}\right\}$.

If all $C_{1}, \ldots, C_{k}$ are homotopic trivial, the theorem is trivial. So assume without loss of generality that $C_{1}$ is not homotopic trivial. Let $e, e^{\prime}$ be the first two edges of $G$ passed by $P_{1}^{1}$. That is, $P_{1}^{1}=\left(v_{0}, e, v_{1}, e^{\prime}, v_{2}, \alpha\right)$ for some string $\alpha$. We consider two cases.

CASE 1. Each of $P_{1}^{1}, \ldots, P_{1}^{K}$ passes $e^{\prime}$ as second edge. In this case no other $P_{i}^{j}$ can pass edge $e^{\prime}$ (by (11)(iii)). Now delete edges $e$ and $e^{\prime}$ from $G$, add a new vertex $w$ in the new face $F^{\prime} \cup e^{\prime} \cup F^{\prime \prime}$ (where $F^{\prime}$ and $F$ are the faces incident to $e^{\prime}$ (possibly $\left.F=F^{\prime}\right)$ ), and add a new edge $e^{\prime \prime}$ connecting $w$ and $v_{2}$. Replace $C_{1}$ by $\left(w, e^{\prime \prime}, v_{2}, \alpha\right)$. Replace $C_{2}, \ldots, C_{k}$ by $P_{2}^{1}, \ldots, P_{k}^{1}$, respectively. Replace $\left\{I_{1}, \ldots, I_{p}\right\}$ by

$$
\left(\left\{I_{1}, \ldots, I_{p}\right\} \backslash\left\{F, F^{\prime}\right\}\right) \cup\left\{\left(F \cup e^{\prime} \cup F^{\prime}\right) \backslash\left(e^{\prime \prime} \cup w\right)\right\} .
$$

We claim that condition (11) is maintained in the new situation. This follows from the fact that in the new situation there exists a "fractional" packing of paths as in the homotopic flow-cut theorem: for each $i=2, \ldots, k$, 
all $P_{i}^{j}$ are homotopic to $P_{i}^{1}$ in $\mathbb{R}^{2} \backslash\left(I_{1} \cup \cdots \cup I_{p} \cup F \cup e^{\prime} \cup F^{\prime}\right)$; moreover, for $j=1, \ldots, K$, we can write $P_{1}^{j}=\left(v_{0}, e, v_{1}, e^{\prime}, v_{2}, \alpha^{\prime}\right)$ so that $\left(w, e^{\prime \prime}, v_{2}, \alpha^{\prime}\right) \sim\left(w, e^{\prime \prime}, v_{2}, \alpha\right)$.

CASE 2. Not each of $P_{1}^{1}, \ldots, P_{1}^{K}$ passes $e^{\prime}$ as second edge. Without loss of generality, path $P_{1}^{2}$ passes edge $e^{\prime \prime} \neq e^{\prime}$ as second edge. Consider the neighbourhood of $v_{1}$, with edges $e_{1}, \ldots, e_{d}$ and faces $F_{1}, \ldots, F_{d}$ as indicated:

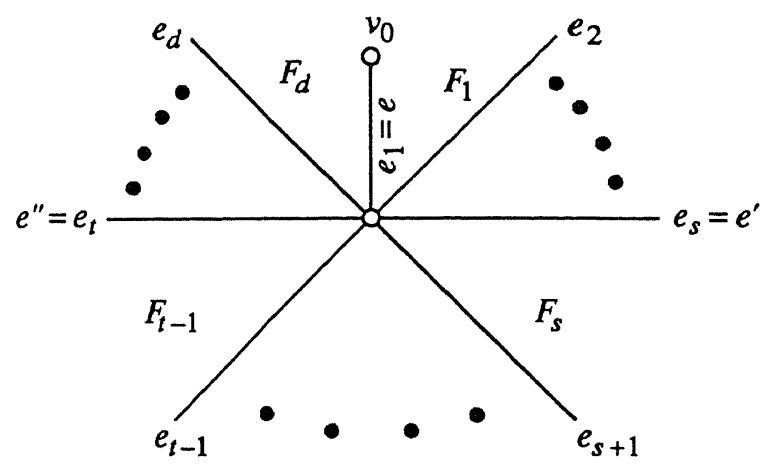

So $e=e_{1}, e^{\prime}=e_{s}, e^{\prime \prime}=e_{t}$, and $F_{1}=F_{d} \in\left\{I_{1}, \ldots, I_{p}\right\}$. As $P_{1}^{1} \cdot\left(P_{2}^{1}\right)^{-1}$ is a homotopic trivial cycle, we know $F_{s}, \ldots, F_{t-1} \notin\left\{I_{1}, \ldots, I_{p}\right\}$. Now let $I_{p+1}:=F_{s}$. We claim

ClaIM 2. For each curve $D:[0,1] \rightarrow \mathbb{R}^{2} \backslash\left(I_{1} \cup \cdots \cup I_{p+1} \cup V\right)$ with $D(0), D(1) \in \operatorname{bd}\left(I_{1} \cup \cdots \cup I_{p+1}\right)$ we have

$$
\operatorname{cr}(G, D) \geq \sum_{i=1}^{k} \min \operatorname{cr}^{\prime}\left(P_{i}^{1}, D\right),
$$

where

$$
\min \operatorname{cr}^{\prime}\left(P_{i}^{1}, D\right):=\min \left\{\operatorname{cr}(\widetilde{P}, \widetilde{D}) \mid \widetilde{P} \sim P_{i}^{1}, \widetilde{D} \sim D \text { in } \mathbb{R}^{2} \backslash\left(I_{1} \cup \cdots \cup I_{p+1}\right)\right\} .
$$

Proof. Let $Q$ be the path from $v_{1}$ to $v_{1}$ following the boundary of face $F_{s}$ clockwise (cf. (15); so $Q$ starts with $e_{s}$ and ends with $e_{s+1}$ ). For $j=1, \ldots, K$, let

$$
\begin{aligned}
& R_{1}^{j}:=P_{1}^{j} \quad \text { if } P_{1}^{j} \text { uses one of the edges } e_{1}, \ldots, e_{s} \text { as second edge; } \\
& R_{1}^{j}:=\left(v_{0}, e, v_{1}, Q, v_{1}, \beta\right) \quad \text { if } P_{i}^{j} \text { uses one of the edges } e_{s+1}, \ldots, e_{d} \text { as } \\
& \text { second edge, and } P_{1}^{j}=\left(v_{0}, e, v_{1}, \beta\right) \text {. }
\end{aligned}
$$

So $R_{1}^{j} \sim P_{1}^{1}$ in $\mathbb{R}^{2} \backslash\left(I_{1} \cup \cdots \cup I_{p+1}\right)$ for $j=1, \ldots, K$. Moreover, for $i=2, \ldots, k$ and $j=1, \ldots, K$ let $R_{i}^{j}:=P_{i}^{j}$. By Claim $1, R_{i}^{j} \sim P_{i}^{1}$ in $\mathbb{R}^{2} \backslash\left(I_{1} \cup \cdots \cup I_{p+1}\right)$, for $i=2, \ldots, k$ and $j=1, \ldots, K$. Now for each 
edge $e$ of $G$ :
(i) $\sum_{i=1}^{k} \sum_{j=1}^{K} \lambda_{i}^{j} \chi^{R_{i}^{j}}(e) \leq 1 \quad$ if $e$ is not incident to $F_{s^{\prime}}$;
(ii) $\sum_{i=1}^{k} \sum_{j=1}^{K} \lambda_{i}^{j} \chi^{R_{i}^{j}}(e)<2 \quad$ if $e$ is incident to $F_{s}$.

(The strict inequalities follow from the fact that the sum of those $\lambda_{1}^{j}$ for which $P_{1}^{j}$ uses one of the edges $e_{s+1}, \ldots, e_{d}$ as second edge is strictly less than 1 (since $P_{1}^{1}$ uses $e_{s}$ as second edge).)

Now choose $D:[0,1] \rightarrow \mathbb{R}^{2} \backslash\left(I_{1} \cup \cdots \cup I_{p+1} \cup V\right)$ with $D(0), D(1) \in$ $\operatorname{bd}\left(I_{1} \cup \cdots \cup I_{p+1}\right)$. We may assume that if $D(z) \in G$, then $D$ has a crossing with $G$ at $z$. This implies that $D \cap \operatorname{bd}\left(F_{s}\right)=\{D(0), D(1)\} \cap \operatorname{bd}\left(F_{s}\right)$.

If not both $D(0)$ and $D(1)$ belong to bd $\left(F_{s}\right)$ we have by (18):

$$
\begin{aligned}
\operatorname{cr}(G, D) & =\sum_{e \in E} \chi^{D}(e)>-1+\sum_{e \in E} \chi^{D}(e) \sum_{i=1}^{k} \sum_{j=1}^{K} \lambda_{i}^{j} \chi^{R_{i}^{j}}(e) \\
& =-1+\sum_{i=1}^{k} \sum_{j=1}^{K} \lambda_{i}^{j} \sum_{e \in E} \chi^{R_{i}^{j}}(e) \chi^{D}(e) \\
& =-1+\sum_{i=1}^{k} \sum_{j=1}^{K} \lambda_{i}^{j} \cdot \operatorname{cr}\left(R_{i}^{j}, D\right) \\
& \geq-1+\sum_{i=1}^{k} \min \operatorname{cr}^{\prime}\left(P_{i}^{1}, D\right) .
\end{aligned}
$$

[Here $\chi^{D}(e)$ denotes the number of times $D$ intersects $e$.] Note that (19) implies (16). If both $D(0)$ and $D(1)$ belong to $\operatorname{bd}\left(F_{s}\right)$, then using (18) one similarly shows

$$
\operatorname{cr}(G, D)>-2+\sum_{i=1}^{k} \min \operatorname{cr}^{\prime}\left(P_{i}^{1}, D\right) .
$$

Now by condition (12)(iii),

$$
\operatorname{cr}(G, D) \equiv \sum_{i=1}^{k} \operatorname{cr}\left(P_{1}^{1}, D\right) \equiv \sum_{i=1}^{k} \min \operatorname{cr}\left(P_{1}^{1}, D\right) \quad(\bmod 2),
$$

since $D(0)$ and $D(1)$ belong to the boundary of the same face $F_{s}$. Now (21) and (20) imply (16).

So by induction there exist pairwise edge-disjoint and pairwise noncrossing paths $\widetilde{P}_{1}, \ldots, \widetilde{P}_{k}$ (without self-crossing and not using the same edge more than once), so that $\widetilde{P}_{i} \sim P_{i}^{1}$ in $\mathbb{R}^{2} \backslash\left(I_{1} \cup \cdots \cup I_{p+1}\right)$, for $i=1, \ldots, k$. This implies $P_{i} \sim P_{i}^{1} \sim C_{i}$ in $\mathbb{R}^{2} \backslash\left(I_{1} \cup \cdots \cup I_{p}\right)$. 


\section{Lemma 1}

The first part of the proof of our theorem consists of showing that conditions (1)(i) and (ii) are equivalent to the existence of a certain "graphdisjoint" system of curves. This is the content of Lemma 1.

Let $G=(V, E)$ be a planar graph embedded in the plane $\mathbb{R}^{2}$, and let $I_{1}, \ldots, I_{p}$ be some of its faces, including the unbounded face. With any curve $C:[0,1] \rightarrow \mathbb{R}^{2}$ we can associate its face sequence

$$
\left(\varphi_{0}, \ldots, \varphi_{t}\right)
$$

where each $\varphi_{j}$ is a vertex, edge, or face of $G$, so that $C$ starts in $\varphi_{0}$, next passes $\varphi_{1}$, next $\varphi_{2}$, and so on, until it terminates in $\varphi_{t}$. (We consider vertices also as a singleton set.) So $\varphi_{j-1}$ and $\varphi_{j}$ are incident for $j=$ $1, \ldots, t .\left(\varphi\right.$ and $\varphi^{\prime}$ are called incident if $\varphi \neq \varphi^{\prime}$ and $\varphi \cup \varphi^{\prime}$ is connected. Now let $C_{1}, \ldots, C_{k}:[0,1] \rightarrow \mathbb{R}^{2} \backslash\left(I_{1} \cup \cdots \cup I_{p}\right)$ be curves, where $C_{i}$ has face sequence

$$
\left(\varphi_{i, 0}, \ldots, \varphi_{i, t_{i}}\right)
$$

for $i=1, \ldots, k$. We call $C_{1}, \ldots, C_{k}$ graph-disjoint (with respect to $\left.G ; I_{1}, \ldots, I_{p}\right)$ if for all $i, i^{\prime}=1, \ldots, k ; j=1, \ldots, t_{i} ; j^{\prime}=1, \ldots, t_{i^{\prime}}$ :

(i) $\varphi_{i, j}=\varphi_{i^{\prime}, j^{\prime}}$ if and only if $i=i^{\prime}$ and $j=j^{\prime}$;

(ii) $\varphi_{i, j}$ and $\varphi_{i^{\prime}, j^{\prime}}$ are incident if and only if $i=i^{\prime}$ and $\left|j-j^{\prime}\right|=1$

(iii) if $i=i^{\prime}$ and $\left|j-j^{\prime}\right|=1$ then each closed curve in $\varphi_{i, j} \cup \varphi_{i, j^{\prime}}$ is homotopically trivial in $\mathbb{R}^{2} \backslash\left(I_{1} \cup \cdots \cup\right.$ $\left.I_{p}\right)$.

Condition (24)(iii) is meant to exclude, e.g., the following situations:

(25)
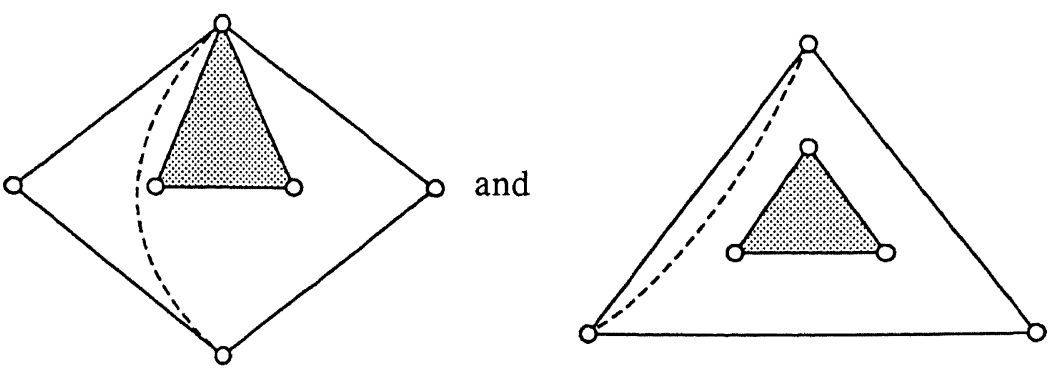

where the interrupted curve indicates a curve $C_{i}$ and where the shaded region 
indicates one of the faces $I_{1}, \ldots, I_{p}$. However, the following is allowed:

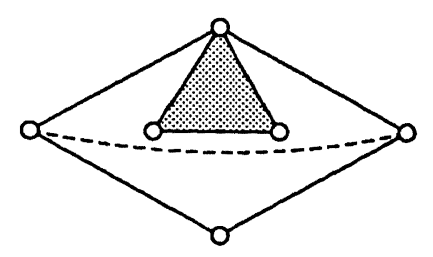

It is easy to see that if each $C_{i}$ is a curve in $G$ (i.e., no element in (23) is a face of $G$ ), then the conditions (24) amount to the $C_{i}$ forming a collection of pairwise vertex-disjoint simple paths in $G$.

We show:

Lemma 1. Let $G=(V, E)$ be a planar graph, embedded in $\mathbb{R}^{2}$, let $I_{1}, \ldots, I_{p}$ be some of its faces (including the unbounded face), and let $P_{1}, \ldots$, $P_{k}$ be paths in $G$, each with end points on $\operatorname{bd}\left(I_{1} \cup \cdots \cup I_{p}\right)$. Then the following are equivalent:

(a) conditions (1)(i) and (ii) hold;

(b) there exists a graph-disjoint collection of curves $C_{1}, \ldots, C_{k}$ where $C_{i} \sim P_{i}$ in $\mathbb{R}^{2} \backslash\left(I_{1} \cup \cdots \cup I_{p}\right)$ for $i=1, \ldots, k$.

Proof. I. To see (b) $\Rightarrow$ (a) in (27), let $C_{1} \sim P_{1}, \ldots, C_{k} \sim P_{k}$ form a graph-disjoint collection of curves. Then clearly, by (24)(i), there are simple curves $\widetilde{C}_{1} \sim C_{1}, \ldots, \widetilde{C}_{k} \sim C_{k}$ which are again graph-disjoint, and hence they are disjoint. This shows (1)(i).

To derive (1)(ii), let $D:[0,1] \rightarrow \mathbb{R}^{2} \backslash\left(I_{1} \cup \cdots \cup I_{p}\right)$ be any curve, with $D(0), D(1) \in \operatorname{bd}\left(I_{1} \cup \cdots \cup I_{p}\right)$, and with face-sequence say $\left(\psi_{0}, \ldots, \psi_{t}\right)$, so that $\operatorname{cr}(G, D)$ is finite. Then $\operatorname{cr}(G, D)=\frac{1}{2}(t+1)$. Moreover, we can draw $D$ so that, leaving its face-sequence and homotopy invariant, it only intersects any $C_{i}$ if it is necessary; that is, $D$ does not intersect any $C_{i}$ both in $\psi_{j-1}$ and in $\psi_{j}$ (as $\psi_{j-1}$ and $\psi_{j}$ are incident, one of them being a face of $G$ ). So

$$
\operatorname{cr}(G, D)=\frac{1}{2}(t+1) \geq \sum_{i=1}^{k} \min \operatorname{cr}\left(C_{i}, D\right),
$$

and therefore (1)(ii) holds.

II. We next show the implication (a) $\Rightarrow$ (b) in (27). To this end, we construct from $G$ an auxiliary graph $G^{\prime}=\left(V^{\prime}, E^{\prime}\right)$ as follows. Let $\varepsilon>0$ be so that $\varepsilon<\frac{1}{2}\|v-w\|$ for each two vertices $v$ and $w$ of $G$. For each vertex $v$ of $G$, remove from $G$ all points in $R_{v}:=\left\{p \in \mathbb{R}^{2} \mid\|p-v\|<\varepsilon\right\}$ and add the circle $S_{v}:=\left\{p \in \mathbb{R}^{2} \mid\|p-v\|=\varepsilon\right\}$. Each vertex $v$ thus gives $\operatorname{deg}(v)$ new vertices, on $S_{v}$. Next, for each edge $e$ of $G$, replace the part of $e$ left by two parallel edges. So, altogether, the neighbourhood of any vertex 
$v$ (for example):

(29)

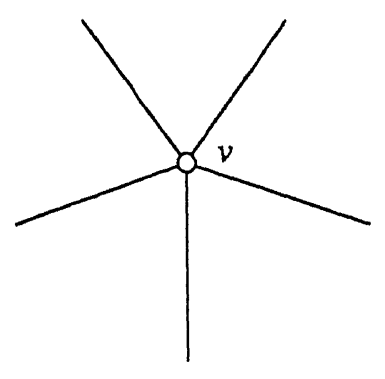

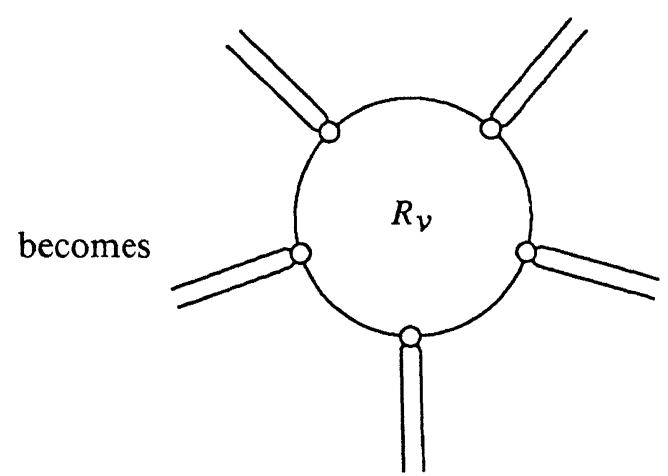

Now if path $P_{i}$ starts in $v \in \operatorname{bd}\left(I_{1} \cup \cdots \cup I_{p}\right)$, choose $j=1, \ldots, p$ so that $v \in \operatorname{bd}\left(I_{j}\right)$. Next add, in the face of the new graph corresponding to $I_{j}$, two new vertices, $v^{\prime}$ and $v^{\prime \prime}$ say, and connect them by edges, say $e_{v^{\prime}}$ and $e_{v^{\prime \prime}}$, to some point on $S_{v} \backslash G$. So

(30)

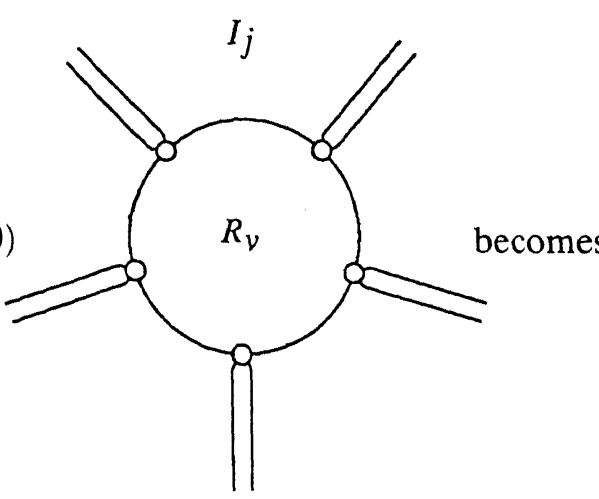

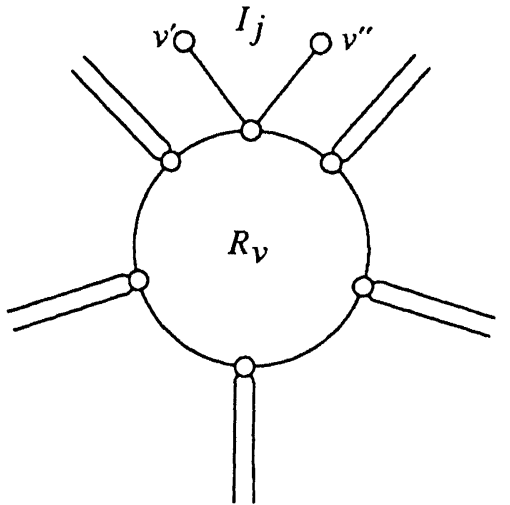

We proceed similarly at the end point $w$ of $P_{i}$, yielding the vertices $w^{\prime}$ and $w^{\prime \prime}$ and edges $e_{w^{\prime}}$ and $e_{w \prime \prime}$. Now replace $P_{i}$ by the curves $P_{i}^{\prime}$ and $P_{i}^{\prime \prime}$ as follows. $P_{i}^{\prime}$ is obtained from $P_{i}$ by adding, at the beginning, a curve from $v^{\prime}$ to $v$, first following $e_{v^{\prime}}$ and next passing $R_{v}$, and at the end, a curve from $w$ to $w^{\prime}$, first passing $R_{w}$ and next following $e_{w^{\prime}}$. Curve $P_{i}^{\prime \prime}$ is obtained similarly from $P_{i}$ using $v^{\prime \prime}, e_{v^{\prime \prime}}, w^{\prime \prime}$, and $e_{w^{\prime \prime}}$.

We do this for each $i=1, \ldots, k$. This defines the graph $G^{\prime}=\left(V^{\prime}, E^{\prime}\right)$, together with the curves $P_{1}^{\prime}, P_{1}^{\prime \prime}, \ldots, P_{k}^{\prime}, P_{k}^{\prime \prime}$. Let $F^{\prime}$ denote the face of $G^{\prime}$ corresponding to any face $F$ of $G$. By condition (1)(i) we know that there exist curves $C_{1}^{\prime} \sim P_{1}^{\prime}, C_{1}^{\prime \prime} \sim P_{1}^{\prime \prime}, \ldots, C_{k}^{\prime} \sim P_{k}^{\prime}, C_{k}^{\prime \prime} \sim P_{k}^{\prime \prime}\left(\right.$ in $\mathbb{R}^{2} \backslash\left(I_{1}^{\prime} \cup \cdots \cup I_{p}^{\prime}\right)$ ) so that $C_{1}^{\prime}, C_{1}^{\prime \prime}, \ldots, C_{k}^{\prime}, C_{k}^{\prime \prime}$ satisfy conditions (12)(i) and (ii) (possibly by flipping $v^{\prime}$ and $v^{\prime \prime}$ in (30)). Clearly, also condition (12)(iii) holds for $G$ 
and $C_{1}^{\prime}, C_{1}^{\prime \prime}, \ldots, C_{k}^{\prime}, C_{k}^{\prime \prime}$. Moreover, condition (1)(ii) implies

$$
\operatorname{cr}\left(G^{\prime}, D^{\prime}\right) \geq \sum_{i=1}^{k} \min \operatorname{cr}\left(C_{i}^{\prime}, D^{\prime}\right)+\sum_{i=1}^{k} \min \operatorname{cr}\left(C_{i}^{\prime \prime}, D^{\prime}\right)
$$

for each curve $D^{\prime}:[0,1] \rightarrow \mathbb{R}^{2} \backslash\left(I_{1}^{\prime} \cup \cdots \cup I_{p}^{\prime} \cup V^{\prime}\right)$ with $D^{\prime}(0), D^{\prime}(1) \in$ $\operatorname{bd}\left(I_{1}^{\prime} \cup \cdots \cup I_{p}^{\prime}\right)$. Indeed, for each such $D^{\prime}$, we can "construct" the parts of $D^{\prime}$ in $\overline{R_{v}}$. We obtain a curve $D$ with $\operatorname{cr}\left(G^{\prime}, D^{\prime}\right)=2 \operatorname{cr}(G, D)$ and $\min \operatorname{cr}\left(C_{i}^{\prime}, D^{\prime}\right) \leq \min \operatorname{cr}\left(P_{i}, D\right), \min \operatorname{cr}\left(C_{i}^{\prime \prime}, D^{\prime}\right) \leq \min \operatorname{cr}\left(P_{i}, D\right)$ for $i=$ $1, \ldots, k$. Hence by (1) (ii) we have (31).

So our auxiliary theorem gives us pairwise edge-disjoint and pairwise noncrossing paths $Q_{1}^{\prime} \sim C_{1}^{\prime}, Q_{1}^{\prime \prime} \sim C_{1}^{\prime \prime}, \ldots, Q_{k}^{\prime} \sim C_{k}^{\prime}, Q_{k}^{\prime \prime} \sim C_{k}^{\prime \prime}$. Let $R_{i}^{\prime}$ and $R_{i}^{\prime \prime}$ be the paths in $G$ obtained from $P_{i}^{\prime}$ and $P_{i}^{\prime \prime}$ by contracting $G^{\prime}$ to $G$, for $i=1, \ldots, k$. Then for each $i=1, \ldots, k$, the cycle $R_{i}^{\prime} \cdot\left(R_{i}^{\prime \prime}\right)^{-1}$ follows the boundary of a simply-connected subset $S_{i}$ of $\mathbb{R}^{2} \backslash\left(I_{1} \cup \cdots \cup I_{p}\right)$ with $P_{i}(0), P_{i}(1) \in S_{i}$, where $S_{i}$ is a union of faces, edges, and vertices. Let $C_{i}$ be a "free-est possible" curve in $S_{i}$ connecting $P_{i}(0)$ and $P_{i}(1)$. This means that if $C_{i}(z)$ is a vertex for some $z$, then there exist $z^{\prime}, z^{\prime \prime} \in[0,1]$ so that $C_{i}(z)=R_{i}^{\prime}\left(z^{\prime}\right)=R_{i}^{\prime \prime}\left(z^{\prime \prime}\right)$ and so that the $0-z^{\prime}$ part of $R_{i}^{\prime}$ is homotopic to the $0-z^{\prime \prime}$ part of $R_{i}^{\prime \prime}$.

Obviously, the curves $C_{1}, \ldots, C_{k}$ form the graph-disjoint system of curves, with $C_{i} \sim P_{i}$ for $i=1, \ldots, k$. This finishes the proof of Lemma 1.

\section{Lemma 2}

The second part of our proof consists of showing that the existence of a graph-disjoint system of curves together with condition (1)(iii) implies the existence of a packing of paths as required by the theorem, which is the content of Lemma 2. So together with Lemma 1 this implies our theorem.

A basic ingredient for the proof of Lemma 2 is the following well-known observation. (For sharpenings, see Deming [2], Sterboul [7], and Korach [5].)

Proposition. Let $G=(V, E)$ be an undirected graph (loops allowed), and let $M \subseteq E$ be a perfect matching. Then $G$ has a coclique $K$ with $|K|=\frac{1}{2}|V|$ if and only if $G$ contains no cycle

$$
\left(v_{0}, e_{1}, v_{1}, \ldots, e_{l}, v_{l}\right)
$$

where

(i) $v_{0}=v_{1}, e_{i}$ is an edge connecting the vertices $v_{i-1}$ and $v_{i}(i=1, \ldots, l)$ and $l$ is even;

(ii) $e_{1}, e_{3}, e_{5}, \ldots, e_{l-1} \in M$ and $e_{2}, e_{4}, \ldots, e_{l} \notin M$;

(iii) $v_{t}=v_{0}$ and $v_{t-1}=v_{1}$ for some odd $t$. 
[Here a loop is considered as a singleton. A perfect matching is a set of $\frac{1}{2}|V|$ edges covering $V$ (so they are pairwise disjoint and nonloops). A coclique is a set of vertices not containing any edge as subset.]

Proof. I. To show the "only if" part, suppose $G$ has a coclique $K$ of size $\frac{1}{2}|V|$ and $G$ contains a cycle (32) satisfying (33). Then for each edge in $M$ exactly one of its end points belongs to $K$. As $v_{0}=v_{l}$ it follows that either $v_{0}, v_{2}, \ldots, v_{l} \in K$ or $v_{1}, v_{3}, \ldots, v_{l-1} \in K$ Since $v_{0}=v_{t}$ and $v_{1}=v_{t-1}$ for some odd $t$, in both cases it follows that $v_{0}, v_{1} \in K$ - a contradiction as $e_{1}$ connects $v_{0}$ and $v_{1}$.

II. The "if" part is shown by induction on $|V|$. Suppose $G$ does not contain any cycle (32) satisfying (33). Then no edge in $M$ has at both of its vertices a loop attached.

If for each edge in $M$, exactly one of its vertices has a loop attached, we can choose for $K$ the set of all vertices at which no loop is attached.

If there exists an edge $e_{0} \in M$ so that at none of its vertices is there a loop attached, let $e_{0}$ connect $v$ and $w$, and define

$$
\begin{aligned}
V^{\prime} & :=V \backslash\{v, w\}, \\
\delta(v) & :=\left\{v^{\prime} \in V^{\prime} \mid\left\{v, v^{\prime}\right\} \in E\right\}, \\
\delta(w) & :=\left\{w^{\prime} \in V^{\prime} \mid\left\{w, w^{\prime}\right\} \in E\right\}, \\
E^{\prime} & :=\left\{e \in E \mid e \subseteq V^{\prime}\right\} \cup\left\{\left\{v^{\prime}, w^{\prime}\right\} \mid v^{\prime} \in \delta(v), w^{\prime} \in \delta(w)\right\}, \\
M^{\prime} & :=M \backslash\left\{e_{0}\right\} .
\end{aligned}
$$

One easily checks that graph $G^{\prime}=\left(V^{\prime}, E^{\prime}\right)$, with perfect matching $M^{\prime}$, again has no cycle (32) satisfying (33). Hence, by induction, $G^{\prime}$ contains a coclique $K^{\prime}$ of size $\frac{1}{2}\left|V^{\prime}\right|$. Then $\delta(v) \cap K^{\prime}=\varnothing$ or $\delta(w) \cap K^{\prime}=\varnothing$ (as $\left\{v^{\prime}, w^{\prime}\right\} \in E^{\prime}$ for each $v^{\prime} \in \delta(v)$ and $\left.w^{\prime} \in \delta(w)\right)$. So $K^{\prime} \cup\{v\}$ or $K^{\prime} \cup\{w\}$ is a coclique of size $\frac{1}{2}|V|$ in $G$.

We derive from this:

Lemma 2. Let $G=(V, E)$ be a planar graph embedded in $\mathbb{R}^{2}$, let $I_{1}, \ldots$, $I_{p}$ be some of its faces (including the unbounded face), and let $P_{1}, \ldots, P_{k}$ be paths in $G$, each with end points on $\operatorname{bd}\left(I_{1} \cup \cdots \cup I_{p}\right)$. Suppose there exists a graph-disjoint system of curves $C_{1} \sim P_{1}, \ldots, C_{k} \sim P_{k}$. If (1)(iii) holds, then there exist pairwise vertex-disjoint simple paths $\widetilde{P}_{1} \sim P_{1}, \ldots, \widetilde{P}_{k} \sim P_{k}$ in $G$.

Proof. From $C_{1}, \ldots, C_{k}$ and $G$ we construct an auxiliary graph $G^{\prime}=$ $\left(V^{\prime}, E^{\prime}\right)$, with a perfect matching $M$, as follows. For $i=1, \ldots, k$, let $C_{i}$ have face sequence

$$
\left(\varphi_{i, 0}, \ldots, \varphi_{i, t_{i}}\right) .
$$

If $\varphi_{i, j}$ is a face of $G$, it is divided by curve $C_{i}$ into two open parts, say $\varphi_{i, j}^{\prime}$ and $\varphi_{i, j}^{\prime \prime}$. Place in each of these parts a point, called $v_{i, j}^{\prime}$ and $v_{i, j}^{\prime \prime}$. All these points (for all $i=1, \ldots, k, j=1, \ldots, t_{i}$ with $\varphi_{i, j}$ a face of $G$ ) form the vertex set $V^{\prime}$ of $G^{\prime}$. 
Let each pair $v_{i, j}^{\prime}, v_{i, j}^{\prime \prime}$ be connected by an edge, drawn in $\varphi_{i, j}$ intersecting $C_{i}$ once. These edges form the perfect matching $M$ in $G^{\prime}$. Moreover, vertices $v_{i, j}^{\alpha}$ and $v_{i^{\prime}, j^{\prime}}^{\beta}$ of $G^{\prime}$ are connected by an edge if:

(i) $i \neq i^{\prime}$ and there exists a vertex $\psi$ of $G$ contained both in $\overline{\psi_{i, j}^{\alpha}}$ and in $\overline{\psi_{i^{\prime}, j^{\prime}}^{\beta}}$; or

(ii) $i=i^{\prime}$, and there exists a vertex $\psi$ of $G$ contained both in $\overline{\varphi_{i, j}^{\alpha}}$ and in $\overline{\varphi_{i^{\prime}, j^{\prime}}^{\beta}}$, so that there is a closed curve $K$, not homotopic trivial in a $\mathbb{R}^{2} \backslash\left(I_{1} \cup \cdots \cup I_{p}\right)$, with face sequence $\left(\psi, \varphi_{i, j}, \ldots, \varphi_{i, j^{\prime}}, \psi\right)$.

Note that (ii) yields a loop in $G^{\prime}$ if $i=i^{\prime}, j=j^{\prime}, \alpha=\beta$, and $\overline{\varphi_{i, j}^{\alpha}}$ contains a closed curve not being homotopic trivial in $\mathbb{R}^{2} \backslash\left(I_{1} \cup \cdots \cup I_{p}\right)$.

In fact, each edge $e$ of $G^{\prime}$ can be represented by a curve in $\mathbb{R}^{2}$ connecting $v_{i, j}^{\alpha}$ and $v_{i^{\prime}, j^{\prime}}^{\beta}$. If $i \neq i^{\prime}$, it starts in $v_{i, j}^{\alpha}$, moves in $\varphi_{i, j}^{\alpha}$ to vertex $\psi$ as in (36)(i), and next moves in $\varphi_{i^{\prime}, j^{\prime}}^{\beta}$ to $v_{i^{\prime}, j^{\prime}}^{\beta}$. If $i=i^{\prime}$, we can make the curve $e$ so that

the closed curve formed by

- the curve $e$,

- the edge in $M$ connecting $v_{i, j}^{\prime}$ and $v_{i, j}^{\prime \prime}$, until its crossing with $C_{i}$,

- the edge in $M$ connecting $v_{i, j^{\prime}}^{\prime}$ and $v_{i, j^{\prime}}^{\prime \prime}$ until its crossing with $C_{i}$,

- the part of $C_{i}$ between these two edges in $M$

is not homotopic trivial in $\mathbb{R}^{2} \backslash\left(I_{1} \cup \cdots \cup I_{p}\right)$.

This defines graph $G^{\prime}=\left(V^{\prime}, E^{\prime}\right)$, with perfect matching $M$, embedded in $\mathbb{R}^{2}$, possibly with crossings. Each edge in $M$ intersects the union of the curves $C_{i}$ exactly once and does not intersect $G$. The edges not in $M$ do not cross any $C_{i}$ and intersect $G$ exactly once.

Now if $G^{\prime}$ has a coclique $K$ of size $\frac{1}{2}\left|V^{\prime}\right|$, then for each $i, j$ exactly one of the two vertices $v_{i, j}^{\prime}$ and $v_{i, j}^{\prime \prime}$ belongs to $K$. Hence for each $i=$ $1, \ldots, k$ :

- the part of the boundary of $\varphi_{i, j}^{\prime}$ not in $C_{i}$, if $v_{i, j}^{\prime} \in$ $K$;

- the part of the boundary of $\varphi_{i, j}^{\prime \prime}$ not in $C_{i}$, if $v_{i, j}^{\prime \prime} \in$ $K$;

- the edges and vertices among $\varphi_{i, 0}, \ldots, \varphi_{i, t_{i}}$ 
contain a simple path $\widetilde{P}_{i} \sim P_{i}-$ in such a way that $\widetilde{P}_{1}, \ldots, \widetilde{P}_{k}$ are pairwise vertex-disjoint. So in this case we are at the required conclusion.

Therefore, assume $G^{\prime}$ has no coclique of size $\frac{1}{2}\left|V^{\prime}\right|$. By the proposition above, it follows that $G^{\prime}$ has a cycle (32) satisfying (33). As $G^{\prime}$ is drawn in $\mathbb{R}^{2}$, we can represent this cycle as a closed curve $D: S_{1} \rightarrow \mathbb{R}^{2}$. Let $D_{1}$ and $D_{2}$ be the closed curves corresponding to parts

$$
\left(v_{0}, e_{1}, v_{1}, \ldots, e_{t}, v_{t}\right) \text { and }\left(v_{t}, e_{t+1}, v_{t+1}, \ldots, e_{l}, v_{l}\right)
$$

of (32). So $D$ can be written as $D_{1} \cdot D_{2}$.

We show that $D_{1}$ and $D_{2}$ give a contradiction to condition (1)(iii). First note that $D_{1}(1)=D_{2}(1)=v_{0}$ does not belong to $G$, as $v_{0}$ is a vertex of $G^{\prime}$. If $D_{1}$ or $D_{2}$ passes vertex $v$ of $G$, then no curve $C_{i}$ passes $v$. So conditions (a) and (b) in (1)(iii) are fulfilled.

Now, $\operatorname{cr}(G, D)$ is equal to the number of $h$ for which $e_{h}$ in (32) does not belong to $M$, while $\sum_{i=1}^{k} \operatorname{cr}\left(C_{i}, D\right)$ is equal to the number of $h$ for which $e_{h}$ belongs to $M$. Hence

$$
\operatorname{cr}(G, D)=\frac{1}{2} l \quad \text { and } \quad \sum_{i=1}^{k} \operatorname{cr}\left(C_{i}, D\right)=\frac{1}{2} l .
$$

Similarly,

$$
\begin{aligned}
& \text { (41) (i) } \operatorname{cr}\left(G, D_{1}\right)=\frac{1}{2}(t-1) \quad \text { and } \quad \sum_{i=1}^{k} \operatorname{cr}\left(C_{i}, D\right)=\frac{1}{2}(t+1) ; \\
& \text { (ii) } \operatorname{cr}\left(G, D_{2}\right)=\frac{1}{2}(l-t+1) \quad \text { and } \sum_{i=1}^{k} \operatorname{cr}\left(C_{i}, D_{2}\right)=\frac{1}{2}(l-t-1) .
\end{aligned}
$$

In particular, by (7), $\operatorname{cr}\left(G, D_{1}\right) \not \equiv \sum_{i=1}^{k} \min \operatorname{cr}\left(P_{i}, D_{1}\right)(\bmod 2)$ and $\operatorname{cr}\left(G, D_{2}\right) \not \equiv \sum_{i=1}^{k} \min \operatorname{cr}\left(P_{i}, D_{2}\right)(\bmod 2)$. So also condition (c) in (1)(iii) is fulfilled.

Now (40) contradicts (1)(iii) when we have proved

$$
\operatorname{cr}\left(C_{i}, D\right)=\min \operatorname{cr}\left(P_{i}, D\right) \quad \text { for } i=1, \ldots, k .
$$

Now $\min \operatorname{cr}\left(P_{i}, D\right)=\min \operatorname{cr}\left(C_{i}, D\right)$ as $P_{i} \sim C_{i}$. By the results of [6], if $\min \operatorname{cr}\left(C_{i}, D\right)<\operatorname{cr}\left(C_{i}, D\right)$, there exist $g, h \in \mathbb{Z}$ so that (taking indices $\bmod l)$ :

(i) $g<h$,

(ii) $C_{i}$ intersects $e_{g}$ and $e_{h}$,

(iii) the part of $C_{i}$ between $e_{g}$ and $e_{h}$ is homotopic to the part $\left(e_{g}, v_{g}, e_{g+1}, v_{g+1}, \ldots, e_{h-1}, v_{h-1}, e_{h}\right)$ of $D^{h-g}$ 
(where $D^{h-g}$ is the closed curve going $h-g$ times around $D$ ). Actually, we begin and end the parts mentioned in (iii) at the crossing points of $e_{g}$ and $e_{h}$ with $C_{i}$.

We may assume that we have chosen $i, g, h$ so that $h-g$ is as small as possible. Note that $h-g$ is even. If $h-g=2$ we are in contradiction with (37). If $h-g>2$, consider the edge $e_{g+2}$. As $e_{g+2} \in M$, there exists an $i^{\prime}$ so that $e_{g+1}$ crosses $C_{i^{\prime}}$ (possibly $i=i^{\prime}$ ). Since the $e_{g}-e_{h}$ part of $C_{i}$ together with $\left(e_{g}, v_{g}, e_{g+1}, v_{g+1}, \ldots, e_{h-1}, v_{h-1}, e_{h}\right)$ forms a homotopic trivial cycle, since $C_{i^{\prime}}$ does not cross $C_{i}$ and since both end points of $C_{i^{\prime}}$ are at one of the faces $I_{1}, \ldots, I_{p}$, there exists an $h^{\prime}$ so that $g+2<h^{\prime}<h$, so that $C_{i^{\prime}}$ crosses $e_{h^{\prime}}$ and so that the $e_{g+2^{-}}-e_{h^{\prime}}$ part of $C_{i^{\prime}}$ is homotopic to $\left(e_{g+2}, v_{g+2}, \ldots, e_{h^{\prime}-1}, v_{h^{\prime}-1}, e_{h^{\prime}}\right)$. As $h^{\prime}-(g+2)<h-g$, this contradicts the minimality of $h-g$.

This completes the proof of Lemma 2.

This completes the proof of our theorem.

REMARK. Note that we in fact proved in Lemma 2 that (if there exists a graph-disjoint set of curves $C_{1} \sim P_{1}, \ldots, C_{k} \sim P_{k}$ ) there exist pairwise vertex-disjoint simple paths $\widetilde{P}_{1} \sim P_{1}, \ldots, \widetilde{P}_{k} \sim P_{k}$ in $G$, if and only if the graph $G^{\prime}$ constructed in the proof has a coclique of size $\frac{1}{2}\left|V^{\prime}\right|$.

\section{Polynomial-time solvability}

It is not directly clear that our theorem gives a "good characterization" for the problem:

$$
\begin{aligned}
\text { given: } & \text { - a planar graph } G=(V, E), \text { embedded in } \mathbb{R}^{2}, \\
& \text { - faces } I_{1}, \ldots, I_{p} \text { of } G \text { (including the unbounded } \\
& \text { face), } \\
& \text { - paths } P_{1}, \ldots, P_{k} \text { in } G \text {, each with end points } \\
& \quad \text { on bd }\left(I_{1} \cup \cdots \cup I_{p}\right), \\
\text { find: } & \text { paths } \widetilde{P}_{1}, \ldots, \widetilde{P}_{k} \text { in } G \text { so that } \widetilde{P}_{i} \sim P_{i} \text { in } \\
& \mathbb{R}^{2} \backslash\left(I_{1} \cup \cdots \cup I_{p}\right) \text { for } i=1, \ldots, k
\end{aligned}
$$

(i.e., that our theorem implies that the decision version of (44) belongs to $\mathscr{N P} \cap$ co- $\mathscr{N P}$ ). We show in this section that problem (44) in fact is solvable in polynomial time (i.e., that (44) belongs to $\mathscr{P}$ ).

We describe a "brute force" polynomial-time method. We do not aim at designing a most efficient algorithm, but rather at giving an existence proof of a polynomial-time method.

We first show that there exists a polynomial-time algorithm for the 
following shortest homotopic path problem:

$$
\begin{aligned}
& \text { given: } \text { - a planar graph } G=(V, E) \text { embedded in } \mathbb{R}^{2}, \\
& \text { - faces } I_{1}, \ldots, I_{p} \text { of } G \text { (including the unbounded } \\
& \text { face), } \\
& \text { - a path } P \text { in } G, \\
& \text { - a "length" function } l: E \rightarrow \mathbb{Z}_{+} ; \\
& \text {find: a path } \widetilde{P} \text { in } G \text { with } \widetilde{P} \sim P \text { in } \mathbb{R}^{2} \backslash\left(I_{1} \cup \cdots \cup I_{p}\right) \text { of } \\
& \\
& \text { shortest length. }
\end{aligned}
$$

[The length of a path $P$ is the sum of the lengths of the edges passed by $P$, counting any edge as often as it is traversed by $P$.]

Proposition 1. Problem (45) is solvable in polynomial time.

Proof. For each pair $i, j \in\{1, \ldots, p\}$ determine a path $Q_{i j}$ in $G$, connecting $\mathrm{bd}\left(I_{i}\right)$ and $\mathrm{bd}\left(I_{j}\right)$, of shortest length. Determine a spanning tree $T$ in the complete graph on $\{1, \ldots, p\}$ of shortest length, where length $(i j):=$ length $\left(Q_{i j}\right)$.

We may assume that if $\{i, j\}$ and $\left\{i^{\prime}, j^{\prime}\right\}$ belong to $T$, then $Q_{i j}$ and $Q_{i^{\prime} j^{\prime}}$ do not cross. Otherwise we could replace $\{i, j\},\left\{i^{\prime}, j^{\prime}\right\}$ either by $\left\{i, i^{\prime}\right\},\left\{j, j^{\prime}\right\}$ or by $\left\{i, j^{\prime}\right\},\left\{j, i^{\prime}\right\}$, without increasing the length of the spanning tree.

To facilitate our description, we "double" each path $Q_{i j}$ with $\{i, j\} \in T$ in the following way:
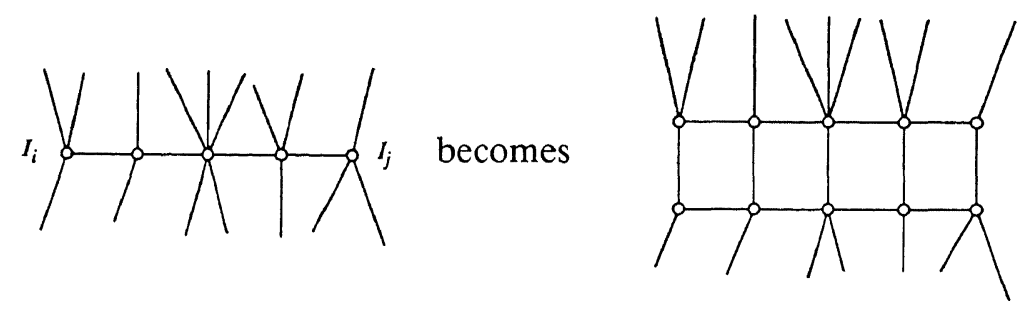

Let $Q_{i j}^{\prime}$ and $Q_{i j}^{\prime \prime}$ denote the two copies of $Q_{i j}$. Let $M_{i j}$ be the matching consisting of the "new" edges connecting $Q_{i j}^{\prime}$ and $Q_{i j}^{\prime \prime}$. Let each edge in $M_{i j}$ have length 0 .

Without loss of generality, our original graph $G$ is of this form. Let $G^{\prime}$ be the graph obtained by deleting all edges in all $M_{i j}$ for $\{i, j\} \in T$. So each circuit in $G^{\prime}$ is homotopic trivial in $\mathbb{R}^{2} \backslash\left(I_{1} \cup \cdots \cup I_{p}\right)$.

Now the homotopy class of any path $R$ in $G$ can be encoded as follows. If $R=\left(v_{0}, e_{1}, v_{1}, \ldots, e_{t}, v_{t}\right)$, we delete from this string the elements $v_{1}, \ldots, v_{t-1}$ and those $e_{g}$ which do not belong to $\bigcup_{\{i, j\} \in T} M_{i j}$. If 
$e_{g} \in M_{i j}$ for some $\{i, j\} \in T$, then

$$
\begin{aligned}
& \text { we replace } e_{g} \text { by } M_{i j} \text { if } v_{g-1} \in Q_{i j}^{\prime} \text { and } v_{g} \in Q_{i j}^{\prime \prime} \text {, and } \\
& \text { we replace } e_{g} \text { by } M_{i j}^{-1} \text { if } v_{g-1} \in Q_{i j}^{\prime \prime} \text { and } v_{g} \in Q_{i j}^{\prime} \text {. }
\end{aligned}
$$

Let us call the string thus obtained the homotopy string of $R$. An example is as follows:

$$
\left(v_{0}, M_{13}, M_{32}^{-1}, M_{57}^{-1}, M_{32}, M_{37}, M_{37}^{-1}, v_{t}\right)
$$

Clearly, this homotopy string determines the homotopy of the path $R$ in $\mathbb{R}^{2} \backslash\left(I_{1} \cup \cdots \cup I_{p}\right)$. Moreover, deleting (repeatedly) any pair of successive symbols $M_{i j}, M_{i j}^{-1}$ or $M_{i j}^{-1}, M_{i j}$, we are left with a string uniquely determined by the homotopy of $R$. Let us call this string the reduced homotopy string of $R$.

Let our input path $P$ have reduced homotopy string $\left(v, \alpha_{1}, \ldots, \alpha_{t}, w\right)$, where $\alpha_{1}, \ldots, \alpha_{t} \in\left\{M_{i j} \mid\{i, j\} \in T\right\} \cup\left\{M_{i j}^{-1} \mid\{i, j\} \in T\right\}$. Now make a graph $H$ as follows. First make $t+1$ copies of $G^{\prime}$, numbered $0,1, \ldots, t$. Next, for $h=1, \ldots, t$, if $\alpha_{h}=M_{i j}$ connect $Q_{i j}^{\prime}$ in the $(h-1)$ th copy of $G^{\prime}$ by a matching (similar to $M_{i j}$ ) to $Q_{i j}^{\prime \prime}$ in the $h$ th copy of $G^{\prime}$. If $\alpha_{h}=M_{i j}^{-1}$ connect $Q_{i j}^{\prime \prime}$ in the $(h-1)$ th copy of $G^{\prime}$ by a similar matching to $Q_{i j}^{\prime}$ in the $h$ th copy of $G^{\prime}$.

The length function $l$ on $G$ can be "lifted" to the edges of $H$ in the obvious way. Let $R$ be a shortest path in $H$ from vertex $v$ in the 0 th copy of $G^{\prime}$ to vertex $w$ in the $t$ th copy of $G^{\prime}$. Let $\tilde{P}$ be the "projection" of $R$ to $G$. We claim that $\widetilde{P}$ is a shortest path homotopic to $P$.

Indeed, let $P^{\prime}$ be a shortest path in $G$ homotopic to $P$. Let $P^{\prime}$ have homotopy string $\left(v, \beta_{1}, \ldots, \beta_{s}, w\right)$. We may assume that no pair of successive elements in this string is equal to $M_{i j}, M_{i j}^{-1}$ : if $M_{i j}, M_{i j}^{-1}$ occurs, we can replace the corresponding part of $P^{\prime}$ by a subpath of $Q_{i j}^{\prime}$ without increasing the length of $P^{\prime}$ (as $Q_{i j}^{\prime}$ is a shortest path) and without changing the homotopy of $P^{\prime}$ (as circuits in $G^{\prime}$ are homotopic trivial). Similarly, we may assume that no two successive elements are equal to $M_{i j}^{-1}, M_{i j}$. But then $P^{\prime}$ is the projection of some path $R^{\prime}$ in $H$ connecting $v$ in the 0 th copy of $G^{\prime}$ with $w$ in the $t$ th copy of $G^{\prime}$. Hence length $\left(P^{\prime}\right)=\operatorname{length}\left(R^{\prime}\right) \geq \operatorname{length}(R)=\operatorname{length}(\tilde{P})$.

Note that the algorithm described also shows that a shortest path $\tilde{P} \sim P$ can be taken so that no edge is passed more than $p \cdot m$ times, where $m$ is the number of edges in $P$ (as the reduced homotopy string of $P$ has at most $p \cdot m$ elements).

We next show that there exists a polynomial-time algorithm for the 
following problem (characterized in our "auxiliary theorem"):

$$
\begin{aligned}
\text { given: } & \text { - a planar graph } G=(V, E), \text { embedded in } \mathbb{R}^{2}, \\
& \text { - faces } I_{1}, \ldots, I_{p} \text { of } G \text { (including the unbounded } \\
& \text { face), } \\
& \text { - curves } C_{1}, \ldots, C_{k} \text {, satisfying (12), } \\
\text { find: } & \text { pairwise edge-disjoint and pairwise noncrossing paths } \\
& P_{1} \sim C_{1}, \ldots, P_{k} \sim C_{k} \text { in } G, \text { without self-crossings } \\
& \text { and not using the same edge more than once. }
\end{aligned}
$$

Proposition 2. There exists a polynomial-time algorithm for problem (49).

Proof. I. We first show that we can decide in polynomial time if paths $P_{1}, \ldots, P_{k}$ exist. In Section 2 above we saw that the existence of these paths is equivalent to the existence of a fractional packing of paths as in the "homotopic flow-cut theorem." This last is equivalent to the fact that the vector $(1, \ldots, 1 ; 1, \ldots, 1) \in \mathbb{R}^{k} \times \mathbb{R}^{E}$ belongs to the convex cone $K$ generated by the following vectors:

$$
\begin{aligned}
& \text { (i) }\left(\varepsilon_{i} ; \chi^{P}\right) \quad\left(i=1, \ldots, k ; P \text { path in } G \text { with } P \sim C_{i}\right) \text {, } \\
& \text { (ii) }\left(\mathbf{0} ; \varepsilon_{e}\right) \quad(e \in E) .
\end{aligned}
$$

Here $\varepsilon_{i}$ denotes the $i$ th unit vector in $\mathbb{R}^{k}$ and $\varepsilon_{e}$ denotes the $e$ th unit vector in $\mathbb{R}^{E}$. Now by the ellipsoid method (see Grötschel, Lovász, and Schrijver [4]), membership of $(1, \ldots, 1 ; 1, \ldots, 1)$ to $K$ can be tested in polynomial time, if for any vector $(d ; l) \in \mathbb{Q}^{k} \times \mathbb{Q}^{E}$ we can test in polynomial time if

$$
(d ; l)(x ; y)^{\mathrm{T}} \geq 0
$$

for every vector $(x ; y) \in K$. This is equivalent to testing if $(d ; l)(x ; y)^{\mathrm{T}} \geq 0$ for every $(x ; y)$ among $(50)$. This last can be done by first testing if $l$ is nonnegative, and if so, by testing, for each $i=1, \ldots, k$ separately, if the minimum length of a path in $G$ homotopic to $C_{i}$ is at least $-d_{i}$. This can be done in polynomial time by Proposition 1.

II. We next show that one actually can find the paths $P_{1}, \ldots, P_{k}$ if they exist. Consider a vertex $v$ of $G$ of degree at least 4 , and "try to" split off two adjacent edges incident to $v$. That is,
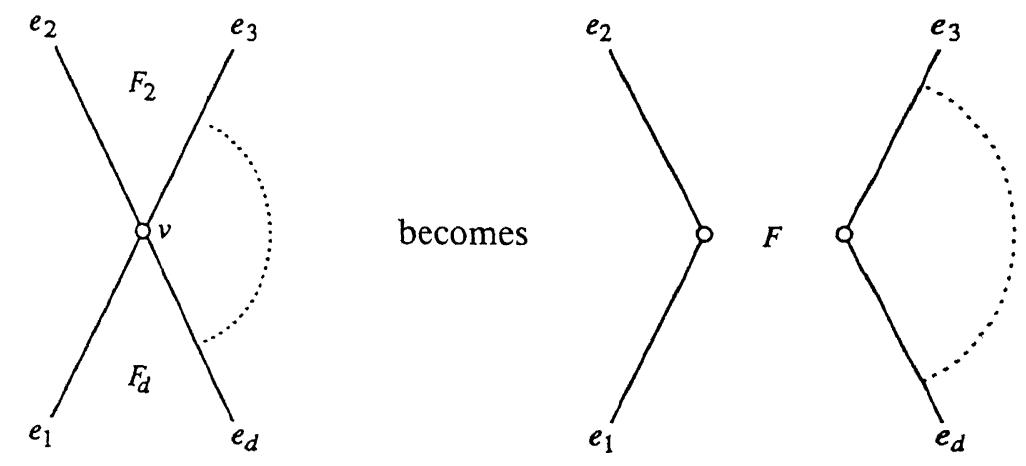
For the new situation, we test if paths $P_{1}, \ldots, P_{k}$ as required exist, where if $F_{2}$ or $F_{d}$ occurs in $\left\{I_{1}, \ldots, I_{p}\right\}$, we replace it by $F$ (see (52)). (If some $C_{i}$ would traverse $F$ we can reroute around the boundary of $F$.) This testing can be done in polynomial time by I above. If these paths exist, we replace $G$ by the new graph. If not, we leave $G$ unchanged.

We do this for each such pair. After at most $|E|^{2}$ iterations, we have a graph in which no more split-offs of such pairs can be performed.

Next for any vertex $v$ of degree at least 6 , and any triple of edges $e_{1}, e_{2}, e_{3}$ incident to $v$ (where $e_{1}$ and $e_{2}$ are adjacent and $e_{2}$ and $e_{3}$ are adjacent), we try to perform a split-off as follows:
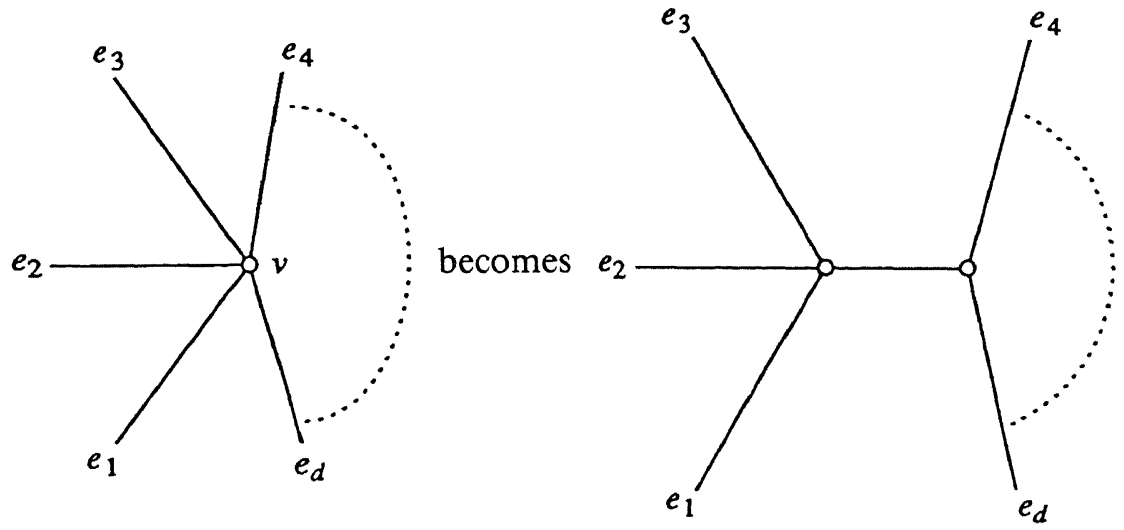

Again, for the new situation we test if paths $P_{1}, \ldots, P_{k}$ as required exist (with I above). If so, we replace $G$ by the new graph. If not, we leave $G$ unchanged.

We do this for each such triple. After at most $|E|^{3}$ iterations, we have a graph in which no more split-offs of such triples can be performed.

As the final graph $G$ contains paths $P_{1}, \ldots, P_{k}$ as required, each vertex $v$ of $G$ has degree at most 4 . For suppose vertex $v$ has degree at least 6 . If no path $P_{i}$ uses vertex $v$, we can split off a pair as in (52). So at least one $P_{i}$ uses vertex $v$. Suppose $P_{i}$ contains $\ldots, e_{1}, v, e_{t}, \ldots$, using notation as in (53). Suppose, moreover, we have chosen $P_{i}$ and the indices of $e_{1}, \ldots, e_{d}$ so that $t$ is as small as possible. If $t=2$ or $t=3$ we could split off a pair or a triple - a contradiction. If $t \geq 4$, then by the minimality of $t$ the edges $e_{2}$ and $e_{3}$ are not used by any $P_{j}$. Hence we could have split off the pair $e_{2}, e_{3}$ from $v$ - a contradiction.

This shows that each vertex of our final graph has degree 1,2 , or 4 . One similarly shows that if one of the paths $P_{i}$ passes a vertex $v$ of degree 4 , 
then it either uses $e_{1}$ and $e_{3}$ or $e_{2}$ and $e_{4}$, using notation given in

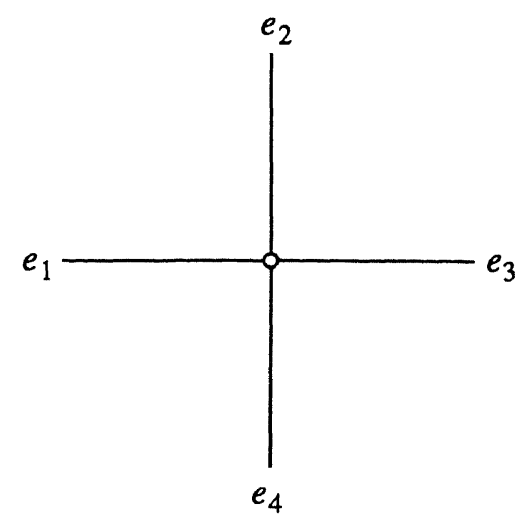

So from our final graph we uniquely determine the paths $P_{1}, \ldots, P_{k}$. This directly yields paths as required in the original graph.

We derive that the problem discussed in Section 3 is solvable in polynomial time:

$$
\begin{aligned}
& \text { given: - a planar graph } G=(V, E) \text {, embedded in } \mathbb{R}^{2} \text {, } \\
& \text { - faces } I_{1}, \ldots, I_{p} \text { of } G \text { (including the unbounded }
\end{aligned}
$$

find: a graph-disjoint collection of curves $C_{1} \sim P_{1}, \ldots$, $C_{k} \sim P_{k}$.

PROPOSITION 3. There exists a polynomial-time algorithm for problem (55).

Proof. We describe a polynomial-time algorithm. Given input as in (55), construct the graph $G^{\prime}$ as in the proof of Lemma 1. By Proposition 2, we can find, in polynomial time, paths $Q_{1}^{\prime}, Q_{1}^{\prime \prime}, \ldots, Q_{k}^{\prime}, Q_{k}^{\prime \prime}$ as in the proof of Lemma 1. Now by contracting $G^{\prime}$ to $G$, we obtain paths $P_{1}^{\prime}, P_{1}^{\prime \prime}, \ldots, P_{k}^{\prime}, P_{k}^{\prime \prime}$. From each pair $P_{i}^{\prime}, P_{i}^{\prime \prime}$ it is not difficult (by following the faces, edges, and vertices at one side of $P_{i}^{\prime}$ ) to identify the face sequence of the curve $C_{i}$, and hence to find $C_{i}$ itself.

Finally we show that our main problem is solvable in polynomial time.

Proposition 4. There exists a polynomial-time algorithm for problem (44).

Proof. We describe a polynomial-time algorithm. Let input as in (44) be given. First find output as in (55) if it exists (with the algorithm of 
Proposition 3). If it does not exist, then neither does output as in (44). If it does exist, construct the graph $G^{\prime}=\left(V^{\prime}, E^{\prime}\right)$ as in the proof of Lemma 2 , together with the perfect matching $M$. Now the existence of paths as required is equivalent to the existence of a coclique of size $\frac{1}{2}\left|V^{\prime}\right|$ in $G^{\prime}$ (see the Remark at the end of Section 4). Now this last can be tested in polynomial time - it is a special case of the 2-satisfiability problem (see Cook [1] and Even, Itai, and Shamir [3]).

Again by a splitting technique as in (52) we can actually find the paths $P_{i}$ as required.

Note that, although our algorithm for (44) uses the ellipsoid method as a subroutine, the final algorithm is "strongly" polynomial: since the input of (44) does not contain numbers, polynomiality and strong polynomiality coincide.

\section{Two examples}

It can be shown that the class of curves $D$ in condition (1)(ii) can be restricted to curves of a simpler type. As an illustration, we close this paper with two examples showing that the closed curves $D_{1}$ and $D_{2}$ can be rather complicated.

In both examples, only one simple path of given homotopy is required, namely that of the straight vertical line connecting vertices $v$ and $w$. The shaded areas indicate the faces in $I_{1}, \ldots, I_{p}$.

Our first example:

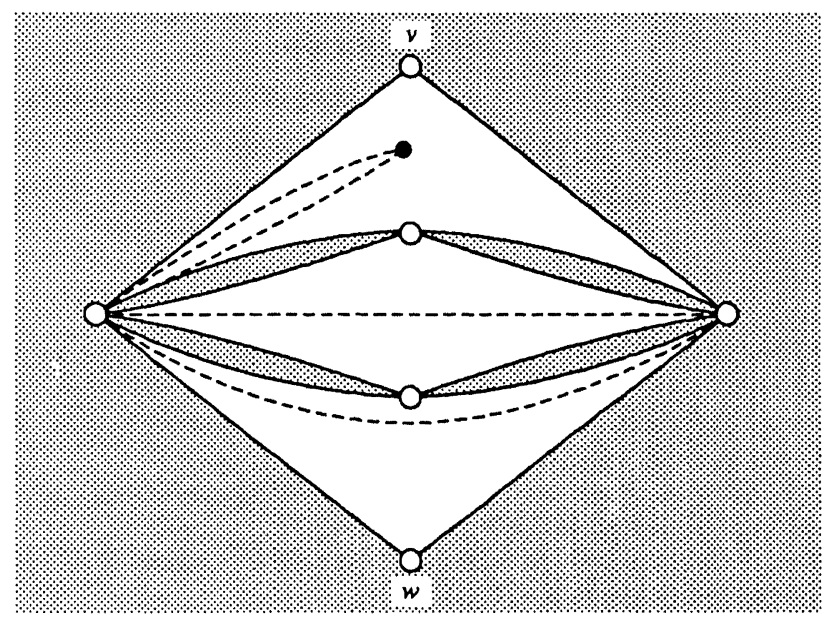


Our second example:

(57)

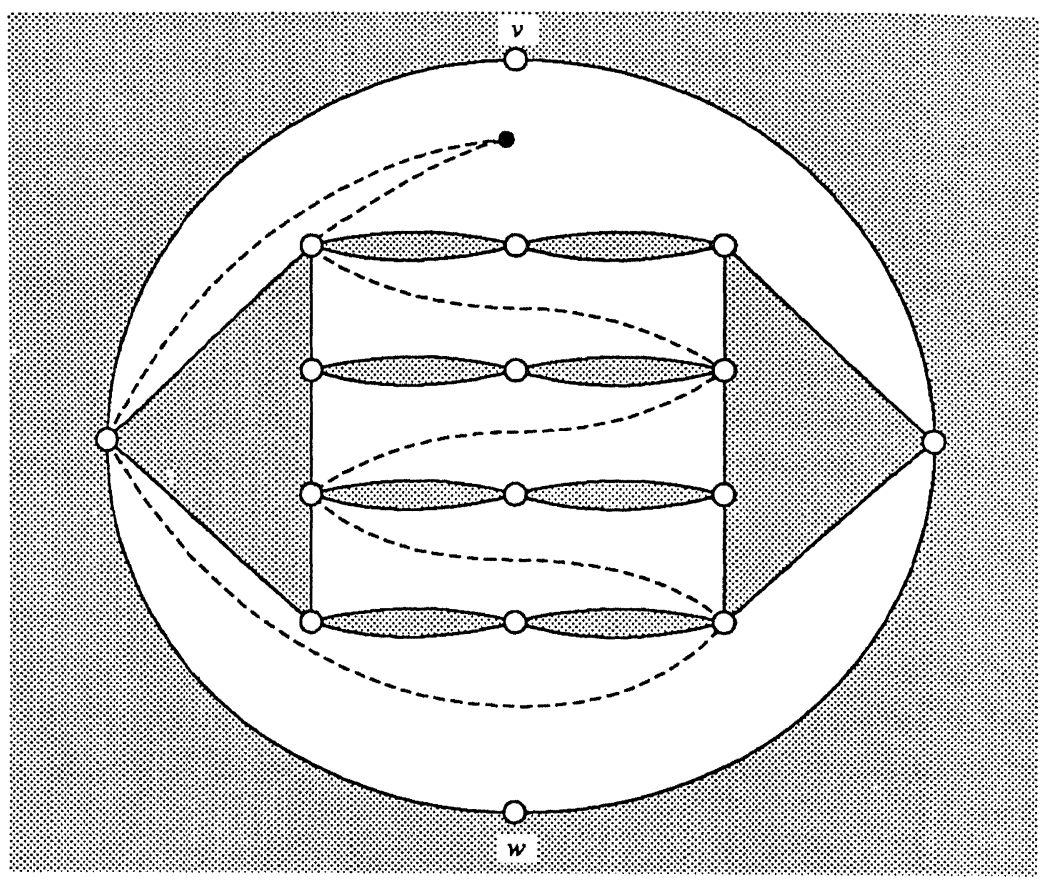

In both examples, conditions (1)(i) and (ii) are satisfied, but there exist closed curves $D_{1}$ and $D_{2}$ violating (1)(iii). Curve $D_{1}$ is indicated by an interrupted curve (where the solid point indicates $D_{1}(1)$ ), while curve $D_{2}$ arises by reflecting $D_{1}$ into the straight line segment $\overline{v w}$.

NoTE. In [7] a combinatorial polynomial-time algorithm for the problem discussed in this paper is given. Moreover, an extension to disjoint homotopic trees is described.

\section{Acknowledgments}

We thank László Lovász and Paul Seymour for very stimulating discussions on the problem discussed in this paper. Moreover, we thank an anonymous referee for carefully reading the text and for helpful suggestions.

\section{REFERENCES}

1. S. A. Cook, The complexity of theorem-proving procedures, in Proceedings of third annual ACM symposium on theory of computing, ACM, New York, 1971, pp. 151-158.

2. R. W. Deming, Independence numbers of graphs-an extension of the Koenig-Egervary theorem, Discrete Math. 27 (1979), 23-33.

3. S. Even, A. Itai, and A. Shamir, On the complexity of timetable and multicommodity flow problems, SIAM J. Comput. 5 (1976), 691-703.

4. M. Grötschel, L. Lovász, and A. Schrijver, Geometric methods and combinatorial optimization, Springer-Verlag, Berlin, 1988.

5. E. Korach, Packing of $T$-cuts and other aspects of dual integrality, $\mathrm{Ph} . \mathrm{D}$. Thesis, University of Waterloo, Waterloo, Ontario, 1982. 
6. A. Schrijver, Decomposition of graphs on surfaces and a homotopic circulation theorem, J. Combin. Theory Ser. B, to appear.

7. _. Disjoint homotopic paths and trees in a planar graph, Discrete Comput. Geom., to appear.

8. F. Sterboul, $A$ characterization of the graphs in which the transversal number equals the matching number, J. Combin. Theory Ser. B 27 (1979), 228-229.

Department of Computer Science, Eötvös Loránd University, Múzeum krt. 6-8, H-1088 BUDAPEST, HUNGARY

Mathematical Centre, Kruislaan 413, 1098 SJ Amsterdam, The Netherlands 